\title{
THE NANOWORKBENCH: Automated Nanorobotic system inside of Scanning Electron or Focused Ion Beam Microscopes.
}

\author{
Ivo Burkart $^{1}$, Eva Burkart ${ }^{1}$, Volker Klocke ${ }^{1}$, David Peters ${ }^{1}$ \\ 1. Klocke Nanotechnik GmbH, Aachen, Germany
}

The Nanoworkbench is the first system substituting eye-hand coordination effectively with nanoprecision in a SEM/FIB-system. It can be imagined how technology could evolve, when tools within a SEM/FIB can be used as easily as tools used under optical microscopes.

Many every day developments would not exist today without preparation, handling and assembly of materials under optical microscopes. There would be no wristwatch, no in vitro fertilization, no minigearbox, just to mention a few. These products depend on using toolsets like tweezers, knives, hooks, probes and several different measurement tools in combination with optical microscopes. But material properties and functionalities also depend on structure dimensions that are smaller than the wavelength of light.

The operators of SEM, FIB or Dual Beam systems generally work without toolsets. One reason for this is the disconnected closed loop operation between human eyes and hands that enable complex operations under optical microscopes without even thinking about it

The two main aspects of the new Nanoworkbech by Klocke Nanotechnik GmbH, the development of its Nanorobotics technology and the applications enabled by it, are described in this paper.

Aspect 1, development of the technology: In general the success of in-SEM/FIB Nanorobotics depends on the co-operation of several important modules in one global system. The main developments include:

- Nanomanipulators in automation, for movement of end-effectors and sample handling,

- Different end-effectors for nano- probing, cutting, cleaning, force distance or wear measurements, gripping, sorting or material preparation and processing,

- $\quad$ Automatic in-situ tip cleaning process

- $\quad$ Automatic 3D position detection of all tools and SEM/FIB

- A control of all tool and SEM/FIB sample stage positions in a common global coordinate system,

- SEM picture assisted haptic interface by "Live Image Positioning",

- Modular design for fast configuration \& teaching of nano-analytical or nano-handling processes.

With instantiating these technical demands the Nanoworkbench enables secure and easy usage of toolsets within SEM/FIB systems, for manual operation, for non-professional users and in high level of automation, e.g. for high throughput industrial processes, even as job-shop [1].

Aspect 2, development of a series of new applications in one system: Expanding the SEM/FIB to a material processing system and a nano-analytical workbench opens the door to many applications in all fields of research and development up to industrial production [5]. Several examples of these new interdisciplinary research and development fields will be described during the presentation.

A few examples of Nanoworkbench applications are highlighted in Figure 1. Although these examples may raise the impression of a review about different machines and their usage, this is not the case. Described is the development of the Nanoworkbench. 
[1] D. Morrant, EIEx Magazine of European Innovation Exchange, 1 (2009)

[2] G. Schmid, M. Noyong, Colloid Polym Sci., (2008)

[3] C.-H. Ke1, H.D. Espinosa, Journal of the Mechanics and Physics of solids, 53 (2005)

[4] Seong Chu Lim, Keun Soo Kim, Kay Hyeok An, Dept. of Phys., Sungkyunkwan University, Korea (2002)

[5] Supported by European Commission, IST and Ziel2.NRW.

Figure 1. includes the rows: 1: Nano-Probing: of Gold55-Clusters arranged in chains [2]: The electrical conductivity along these gold chains is measured over different distances and compared with the conductivity of bare gold wires. 2: Nano-Tribology and similar measurements can be performed in high resolution [4] 3: Nano-Cutting: fast milling of structures 4: 3D-Nanofinger: measurement of gold and EBID structures grown and measured in situ 5: Particle-Sorting: Gripping of a rigid CNT bundle with high force and separation from the ground [3]

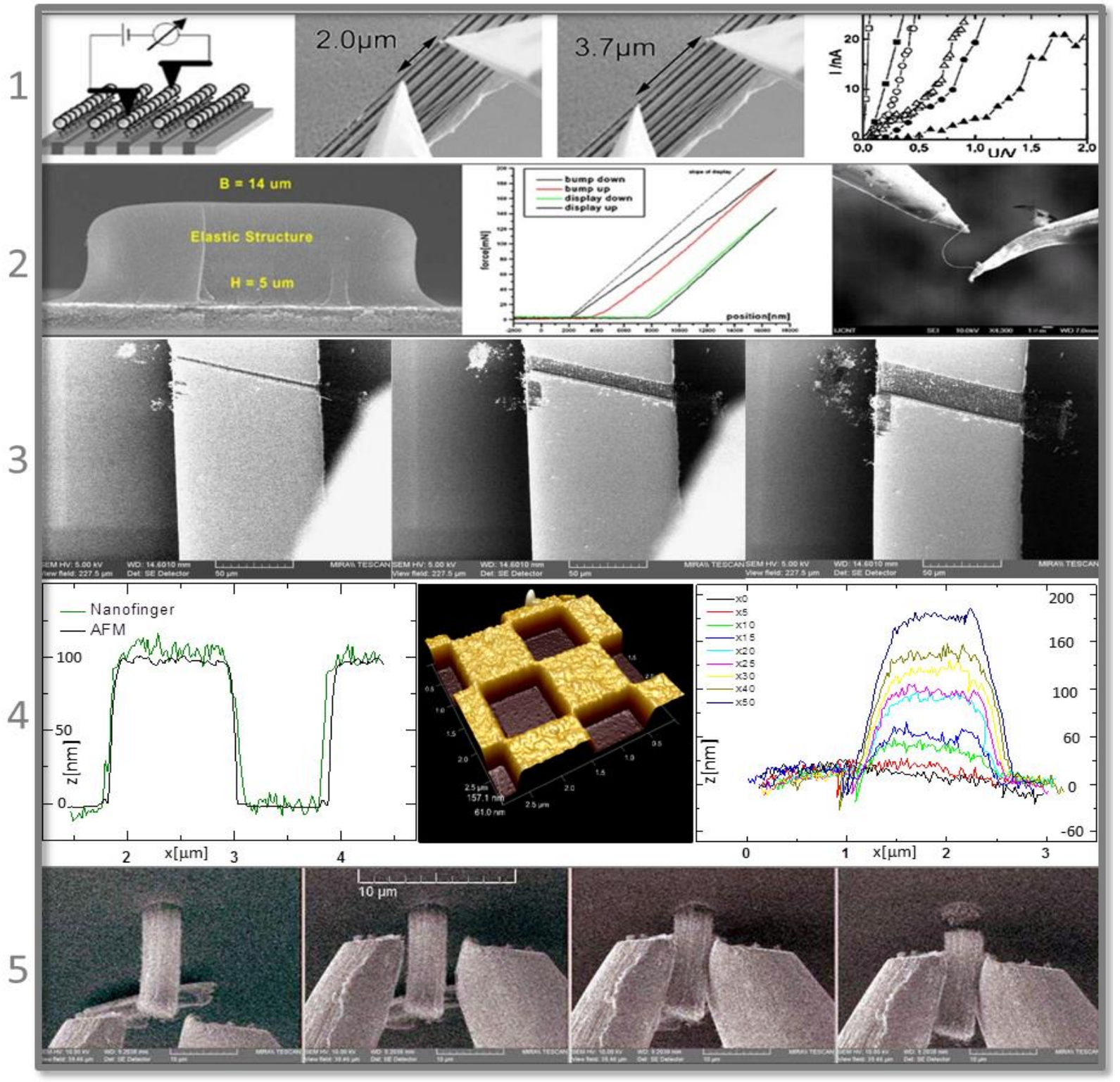

